\title{
José Enrique Rodó oder Shakespeare, Santa Teresa und Zarathustra in Amerika
}

Kommen wir zum Schluss unserer Vorlesung zu jenem schmalen Bändchen, das zumindest aus heutiger Sicht das 19. Jahrhundert abschließt, das 20. Jahrhundert wie einen Paukenschlag eröffnet und nach anfänglichem Schweigen die literarischen und kulturtheoretischen Debatten über Lateinamerika für nahezu ein Vierteljahrhundert entscheidend mitprägen sollte: José Enrique Rodós mit dem neuen Jahrhundert erschienener Band Ariel. Sein großes Verdienst war es, auf diesen Seiten eine Vision für das lateinische Amerika entfaltet zu haben:

Werdet Ihr es nicht sehen, das Amerika, das wir uns erträumen, gastfreundlich zu den Dingen des Geistes und nicht nur zu jenen Menschenmassen, die bei ihm Schutz suchen, gedankenvoll, ohne Minderung seines Talents zur Tat, heiter und fest, trotz seiner großherzigen Begeisterungsfähigkeit, erstrahlend im Zauber eines frühen und sanften Ernstes, wie er den Ausdruck eines kindlichen Gesichts verschönt, wenn sich in ihm mit der aufblitzenden, unberührten Grazie das unruhige, erwachende Denken offenbart? ...- ${ }^{1}$

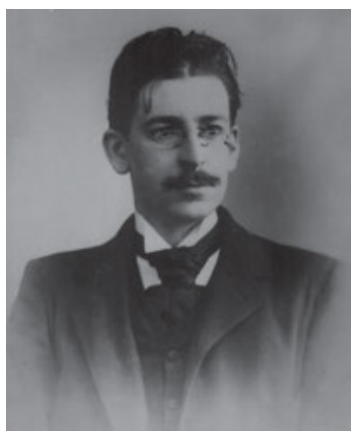

Abb. 87: José Enrique Rodó

(Montevideo, 1871 - Palermo, Italien, 1917).

Diese Vision des kommenden, des bereits entstehenden Amerika leitet den Schlussteil von Prósperos Rede an seine Schüler in José Enrique Rodós sicherlich einflussreichstem Werk ein, das in Vernachlässigung eines anderen Hauptwerks im Mittelpunkt dieses abschließenden Kapitels stehen soll. ${ }^{2}$ In ihr entwirft der

1 Rodó, José Enrique: Ariel. Übersetzt, herausgegeben und erläutert von Ottmar Ette. Mainz: Dieterich'sche Verlagsbuchhandlung 1994, S. 178.

2 Vgl. zu dem faszinierenden, aber weniger im Fokus der Öffentlichkeit stehenden Opus Magnum Rodós Ette, Ottmar: Archipelisches Schreiben und Konvivenz. José Enrique Rodó und seine 
„viejo y venerado maestro“3 das Bild eines Kontinents, dessen Gastfreundschaft nicht nur für die Menschenmassen der Einwanderer gelten dürfe, sondern sich vor allem auf die Dinge des Geistes beziehen müsse. Die Metaphorik der Gastfreundschaft bindet in dieser Passage des im Jahre 1900 erschienenen Bändchens zwei verschiedene historische Entwicklungen zusammen: Zum einen ist dies die Einwanderungswelle, welche wesentlich dazu beitrug, dass sich die Bevölkerung in den lateinamerikanischen Staaten zwischen 1850 und 1900 verdoppelte; ${ }^{4}$ und zum anderen der Prozess der Internationalisierung von Kultur und Literatur in Lateinamerika. Es handelt sich dabei um einen Prozess, der sich im Kontext der dritten Phase beschleunigter Globalisierung im letzten Drittel des 19. Jahrhunderts intensiviert hatte und durch den hispanoamerikanischen Modernismo seine ästhetisch-literarische Ausdrucksform erhielt.

Sie entnehmen dieser statistischen Aufschlüsselung - die freilich nur approximative Werte enthält, wurden doch etwa in Argentinien bis Ende des 19. Jahrhunderts die indigenen Bevölkerungen nicht in die Bevölkerungsstatistiken aufgenommen -, dass die Bevölkerungsentwicklung in Hispanoamerika überaus rapide verlief, was noch deutlicher würde, wenn man den beobachteten Zeitraum noch genauer unterteilte. Dann sähe man, dass die gezielte Einwanderungspolitik vor allem im letzten Drittel des 19. Jahrhunderts insbesondere in den Küstenräumen sehr erfolgreich war und eine enorme Beschleunigung aller sozioökonomischen Modernisierungsprozesse auslöste. Während die Länder mit großem atlantischem Küstenanteil boomten (so etwa Argentinien), gerieten im Verhältnis dazu die lateinamerikanischen Länder, die das Innere des Subkontinents ausmachen (wie etwa Bolivien und Paraguay) ins Hintertreffen. Während die Bevölkerung Argentiniens um fast 1600 Prozent wuchs, tat dies die bolivianische nur um etwa 160 Prozent, das heißt zehnmal weniger. Brasilien siedelt sich zwischen beiden Extremen an mit einer Erhöhung der registrierten Bevölkerungszahl um etwas mehr als 700 Prozent. Aufschlussreich ist, dass der Bereich des Río de la Plata

„Motivos de Proteo“. In: Romanistische Zeitschrift für Literaturgeschichte / Cahiers d'Histoire des Littératures Romanes (Heidelberg) XLII, 1-2 (2018), S. 173-201.

3 Rodó, José Enrique: Ariel. Motivos de Proteo. Prólogo: Carlos Real de Azúa. Edición y Cronología Angel Rama. Caracas: Biblioteca Ayacucho 1976, S. 3.

4 Eine Verdoppelung der Bevölkerungszahl lässt sich auch für die Zeit zwischen 1870 und 1914 - mit einer leichten Verschiebung die Zeitspanne des Modernismo wie der dritten Phase beschleunigter Globalisierung - errechnen. Das von Hans-Jürgen Ille im Rahmen eines VW-Projekts vorgelegte Zahlenmaterial ist in die Endredaktion des von Hans-Otto Dill, Carola Gründler, Inke Gunia und Klaus Meyer-Minnemann herausgegebenen Bandes Apropiaciones de realidad en la novela hispanoamericana de los siglos XIX y XX. Frankfurt am Main: Vervuert 1994 leider nicht aufgenommen worden. 
über den Verlauf des 19. Jahrhunderts hinweg eine prozentual noch höhere Explosion der Bevölkerung durch Zuwanderung verzeichnen konnte als selbst die Vereinigten Staaten von Amerika: Dies mag Ihnen einen Eindruck geben von jenen Entwicklungen, auf die auch in Rodós Ariel angespielt wird.

In den Worten des alten Lehrmeisters Próspero zeichnet sich ein kindliches Antlitz ab, in dessen Gesichtszügen sich die kommende Zeit, die anbrechende Epoche spiegelt. ${ }^{5}$ Damit ist keineswegs eine simple Wiederaufnahme der metaphorischen Rede vom jungen Kontinent verbunden, welche ein wichtiges Element ebenso des kollektiven Selbst- wie Fremdbilds Lateinamerikas im 19. Jahrhundert darstellte - wird doch in Prósperos Rede Jugendlichkeit ja gerade als vorrangig kulturelles und nicht biologisches Merkmal in heidnischer Antike und christlicher Heilslehre gedeutet und dialektisch für die Zukunft des Subkontinents entfaltet. Es erfolgt vielmehr eine Abgrenzung gegenüber diesem zurückliegenden Jahrhundert, das der alte Próspero in seiner Abschlussrede ein letztes Mal auf die Dinge hin befragt, die es in positiver Weise an die nun beginnende Zeit weitergeben könne. ${ }^{6}$ Das an die Schüler vermittelte Zeitbewusstsein ist das eines überwundenen Fin de siècle, einer mit dem neuen Jahrhundert anbrechenden Ära, die in der Bewegung des zum Fluge ansetzenden Ariel symbolische Gestalt annimmt: Ariels Bronzestatue beherrscht den Raum, in welchem Prosperos Schüler ein letztes Mal den Worten des Meisters lauschen, bevor sie ihren eigenen Flug in eine neue Zeit beginnen.

Dieses Bewusstsein, am Beginn eines neuen Zeit-Raums, eines Aufbruchs in die Moderne zu stehen, soll im Folgenden zum einen auf die Zusammenhänge bezogen werden, die zwischen dem sozioökonomischen Begriff der „Modernización“, dem epochenspezifischen Begriff der „Modernidad“ und dem literarästhetischen Begriff des „Modernismo“ bestehen. Zum anderen soll gefragt werden, auf welche Weise diese drei genannten Entwicklungskomplexe literarisch in dem wohl bekanntesten und einflussreichsten Werk des uruguayischen Schriftstellers vermittelt sind. Im Fokus stehen dabei jene intertextuellen Beziehungen, welche die Literaturen des 19. Jahrhunderts einmal mehr im Spannungsfeld zwischen zwei Welten zeigen und dabei die literarischen Bezugssysteme ebenso auf William Shakespeare wie auf den für das Fin de siècle grundlegenden Denker Friedrich Nietzsche orientieren.

5 Auf die sakrale Dimension dieser Verkündigung des kommenden Gottes habe ich hingewiesen in meinem Nachwort zu Rodó, José Enrique: Ariel.

6 Vgl. zur Definition des Modernismus Rodó, José Enrique: Rubén Darío. Su personalidad literaria, su última obra. In (ders.): Obras Completas. Editadas con introducción por Emir Rodríguez Monegal. Madrid: Aguilar 1957, S. 187. 
An erster Stelle soll dabei die Untersuchung von Raum- und Bewegungsstrukturen stehen, welche die ästhetisch überzeugende Umsetzung der skizzierten transarealen Problematik in Rodós Ariel erlauben. Sie weisen diesem längst klassisch gewordenen Text des hispanoamerikanischen Modernismo eine erneuerte Bedeutung im Horizont von Fragen zu, welche auf in Lateinamerika entwickelte divergierende Moderne-Konzepte zielen. Denn während der zurückliegenden Jahrzehnte haben literatur- und kulturtheoretische Ansätze in Lateinamerika Heterogenität und Hybridisierung als Charakteristika einer peripheren Modernität des Subkontinents ausgemacht. ${ }^{7}$ Besonders die letztgenannte, von Beatriz Sarlo entwickelte Begriffsprägung ${ }^{8}$ hat sich als fruchtbar erwiesen, um die eigene, aber zugleich an die hegemonialen Zentren außerhalb Lateinamerikas rückgekoppelte kulturelle Entwicklung zu verdeutlichen und ihren Charakter als „modernización dependiente y desigual“9 herauszuarbeiten. Freilich erscheint im literarischen Bereich die Abhängigkeit und Dependenz Amerikas aus heutiger Sicht als weniger ausgeprägt und hat dem Begriff einer divergierenden Moderne jenseits einer Unterscheidung von ,Zentren' und ,Peripherien' Platz gemacht.

Insbesondere im letzten Drittel des 19. Jahrhunderts zeichneten sich Entwicklungen ab, die im sozioökonomischen Bereich durch eine von Europa und den USA ausgehende Fremdsteuerung des Entwicklungsprozesses, eine wachsende kapitalistische Durchdringung und einen rasch zunehmenden Einbau Lateinamerikas in weltwirtschaftliche Prozesse geprägt waren. Im kulturellen Bereich lässt sich eine steigende Alphabetisierungsrate, der Aufbau eines lateinamerikanischen Verlagswesens und die Schaffung eines eigenen, wenn auch bescheidenen Markts für nationale Druckerzeugnisse nachverfolgen. Der literarische Bereich schließlich erfuhr die Ausdifferenzierung eines literarischen Feldes, die Veränderung der sozialen Funktion des Schriftstellers und die Zunahme der Kommunikationen zwischen den einzelnen literarischen Zentren Lateinamerikas. Innerhalb dieser hier nur schlaglichtartig zu beleuchtenden Entwicklung wuchsen der Literatur in Lateinamerika neue Aufgaben und Funktionen zu, entzogen doch die von der „Modernización“ ausgelösten Säkularisierungsprozesse zusammen mit den Immigrationswellen den alten Institutionen und ihren Werten - betroffen waren

7 Zum Beginn dieser Diskussionen vgl. etwa Rincón, Carlos: Modernidad periférica y el desafío de lo postmoderno: perspectivas del arte narrativo latinoamericano. In: Revista de crítica literaria latinoamericana (Lima) XV, 29 (1989), S. 61-104; vgl. auch Scharlau, Birgit / Münzel, Mark (Hg.): „Kulturelle Heterogenität“ in Lateinamerika. Bibliographie mit Kommentaren. Tübingen: Narr 1991. 8 Vgl. Sarlo, Beatriz: Una modernidad periférica: Buenos Aires 1920 y 1930. Buenos Aires: Ediciones Nueva Visión 1988.

9 Ramos, Julio: Desencuentros de la modernidad en América Latina. Literatura y política en el siglo XIX. México: Fondo de Cultura Económica 1989, S. 12. 
vor allem Klerus und einheimische Oligarchie - zunehmend die Legitimationsbasis. ${ }^{10}$ Damit bot sich den Schriftstellerinnen und Schriftstellern zum einen die Chance, die Literatur zum Ort der Diskussion über jene Werte zu machen, die für die gesamtgesellschaftliche Entwicklung richtungsweisend sein sollten. Zum anderen räumte eine Literatur, die ihre soziale Orientierungsfunktion aus einer neuen Beziehung zum intellektuellen Feld und vor allem zum Feld der Macht ableitete, dem Schriftsteller selbst nun die Möglichkeit ein, vom Feld der Literatur aus nicht nur auf politische Prozesse Einfluss zu nehmen, sondern gleichsam zum Vordenker künftiger gesellschaftlicher Entwicklungen zu werden. José Enrique Rodó ist trotz (oder gerade wegen) seiner politischen Aktivitäten ein hervorragendes Beispiel für diese Entwicklung: Mit seiner Tätigkeit als Abgeordneter im uruguayischen Repräsentantenhaus erreichte er zu keinem Zeitpunkt jene Breitenwirkung, die er mit den wenigen aber substanzreichen, den „pocas pero sustanciosas páginas“11 (so Leopoldo Alas in seiner positiven Besprechung von 1900) seines Ariel erzielte. Die Leitfunktion literarischer Artefakte ist daher für diesen Zeitraum evident.

Raum- und Bewegungsstrukturen beherrschen von Beginn an dieses Hauptwerk des Rodó'schen Modernismo, das sich ebenso dezidiert wie José Martís Nuestra América von der Stimmung des Fin de siècle abwendet. Das erste Kapitel dient dazu, die verschiedenen Personen des Textes einzuführen - Próspero, die Bronzestatue Ariels, die Schüler sowie die Bücher, die als die Getreuen, die „fieles compañeros “12 des Lehrmeisters vorgestellt werden - und die sich anschließende (Sprach-)Handlung zu situieren. Diese gestaltet sich wie folgt: Prósperos Schüler kommen am Ende eines Studienjahres ein letztes Mal in einer weiten Aula ${ }^{13}$ zusammen, die halb Lehrsaal, halb Bibliothek ist und von der Bronzestatue des Shakespeare'schen Luftgeistes zentriert wird. Erst im achten und letzten Kapitel werden die Schüler diesen Saal wieder verlassen und hinaustreten in einen urbanen, großstädtischen Raum, der seinerseits eingebettet ist in kosmische Dimensionen. Der Kontakt mit der Masse, der ,áspero contacto de la muchedumbre“, ${ }^{14}$ führt sie in die Realität (,realidad“) zurück, die aber gleichsam über-

10 Vgl. Gutiérrez Girardot, Rafael: La literatura hispanoamericana de fin de siglo. In: Iñigo Madrigal, Luis (Hg.): Historia de la Literatura Hispanoamericana. Bd. II: Del neoclasicismo al modernismo. Madrid: Ediciones Cátedra 1987, S. 497-506.

11 Alas (Clarín), Leopoldo: Ariel. Hier zitiert nach der von Hugo D. Barbagelata herausgegebenen Sammlung Rodó y sus Críticos. Paris: Imprimerie de Mr Vertongen 1920, S. 42.

12 Rodó, José Enrique: Ariel. Motivos de Proteo, S. 3.

13 Ebda.

14 Ebda., S. 55. 
höht wird durch den Sternenraum, dessen Vibrationen die sakrale Atmosphäre der Rede Prósperos ins Kosmische ausweiten.

Kapitel I und VIII verorten damit das Geschehen in einem einzigen Raum, der sich erst am Ende auf die (lateinamerikanische) Großstadt und den Kosmos hin öffnet. Gleichzeitig übernehmen diese beiden Textteile die Funktion einer fiktionalen Rahmung der Kapitel II bis VII, indem eine auktorial modellierte Erzählerfigur nicht nur die Raumstruktur einführt, sondern auch durch die Ekphrasis der Statue dem Luftgeist Shakespeares plastische Präsenz verleiht. Die symbolische Gegensätzlichkeit zwischen Ariel und Calibán wird in Anschluss an die aus William Shakespeares The Tempest übernommene Personenkonstellation (Próspero - Ariel - Calibán) und mehr noch an deren Deutung in Ernest Renans Caliban entwickelt. Sie erscheint durch die Vermittlung der Erzählerfigur zunächst in sehr schematischer, bipolarer Form; in der Gestaltung der „sala de estudio“ aber fehlt alles, was Calibán repräsentieren könnte. Der Sohn der Sycorax ist aus diesem Raum ebenso ausgeschlossen wie ,die Realität‘ der Menschenmassen, die Lehrsaal und Bibliothek gleichwohl umgibt. Damit präsentiert dieser Raum eine keineswegs bipolare, sondern eine vielmehr auf Ariel zentrierte homogene Struktur. Diese wird noch dadurch verstärkt, dass Próspero am Anfang und am Ende seiner Rede Ariel als Schutzgottheit in den Mittelpunkt stellt und durch Berührung der Stirn des „airy spirit“ letztlich nur in der Rolle eines Propheten erscheint, der von einer in der Statue symbolisierten göttlichen Kraft erleuchtet wird: Ariel steht im Zentrum.

Die Erzählerfigur des ersten und achten Kapitels, die keinesfalls mit dem Autor gleichgesetzt werden darf, bewirkt eine fiktionale Fundierung des gesamten Textes. Damit ist die Rede Prósperos nicht mehr als selbständiger Text aufzufassen: Sie erweist sich vielmehr als Binnenstrukturierung, als eingebettete literarische Form. Dies bedeutet zugleich, dass die bislang vorherrschende Bezeichnung Ariels als Essay einer dringenden Revision bedarf, handelt es sich bei diesem Text in seiner Gesamtheit doch keineswegs um nicht-fiktionale und nicht-erzählende Prosa, sondern vielmehr um eine Mischung fiktionaler und diktionaler Textsorten, die wir als Friktion bezeichnen dürfen. Weder Próspero noch Erzähler dürfen als textinterne Instanzen mit dem außerhalb des literarischen Textes befindlichen Autor José Enrique Rodó identifiziert werden. Auf einer solchen Identifikation Rodós mit Próspero, Erzählerfigur und sogar Ariel aber beruhte ein Gutteil der großen Wirkung, die der Text seit seinem Erscheinen zu Beginn eines neuen Jahrhunderts entfaltete. Damit stellt sich die Frage, welche textexternen und textinternen Faktoren eine solche Rezeption begünstigten, die bis heute noch vorzuherrschen scheint. ${ }^{15}$

15 Vgl. etwa die bemerkenswert eurozentrischen Ausführungen in Volger, Gernot: Mestizenkultur. Lateinamerikas Identität im Spiegel seines zeitgenössischen Denkens. In: Merkur (München) 
Wir müssen die räumlichen Dimensionen des Textes noch präziser erfassen! Die Grundstruktur Ariels weist eine fiktionale Rahmung auf, innerhalb derer die Rede Prósperos angesiedelt ist. Innerhalb dieses literarischen Genres der Rede, die sich insbesondere an der Form der Universitätsreden in der Area des Río de la Plata orientierte, ${ }^{16}$ sind andere Gattungen und Gattungselemente angesiedelt. Auf dieser architextuellen Ebene wären Bestandteile etwa des philosophisch-literarischen Dialogs zu nennen, welche vor allem auf die Dialogues philosophiques Renans oder die Dialoge Platons zurückverweisen, auf die im Text explizit wie implizit angespielt wird; daneben lassen sich aber auch Elemente des Hymnus, des Bühnenmonologs, der literarischen Kritik oder des wissenschaftlichen Traktats ausmachen. Die eingeblendeten Gattungen betreffen vor allem den Essay, wobei die in dessen lateinamerikanischer Variante so häufig zu konstatierende Wichtigkeit oratorischer Elemente eine Verschmelzung rhetorischer und essayistischer Formen in Prósperos Worten in besonderer Weise begünstigte. Einen Eindruck hiervon hatten wir bereits durch Martís Essay Nuestra América erhalten. Darüber hinaus finden aber auch narrative Formen Aufnahme, denen etwa die Erzählung vom „Gastfreundlichen König“ oder von der ihren Bräutigam erwartenden Braut zuzurechnen sind. Auf Ebene der Rede sind diese narrativen Formen ganz im Sinne der antiken Rhetorik präzisen Orten und Funktionen zugewiesen, dienen sie doch - wie der Redner Próspero stets betont - der Verdeutlichung und Veranschaulichung bestimmter oratorischer Argumente.

Die Mischung verschiedenster Gattungselemente in Rodós Text verbietet ebenso wie dessen fiktionale Fundierung eine einfache Zuordnung Ariels zu einem bestimmten Genre; doch wirkt diese Vielfalt nur auf den ersten Blick verwirrend. Denn sie ist eingebunden in eine strenge Hierarchie, die den Text auf der architextuellen Ebene keineswegs als Patchwork auszeichnet, sondern vielmehr als einen Bewegungs-Raum ausweist, der sich gleichsam von außen nach innen hin auf verschiedenen Ebenen strukturiert: Umgeben von einem äußeren Bereich fiktionaler Rahmung siedelt sich die Rede Prósperos an, innerhalb derer nach den Regeln einer bestimmten Dispositio Gattungsformen angeordnet sind, die einerseits funktional auf die Rede wie auf den Rahmen bezogen werden, andererseits aber auch wiederum Elemente anderer Gattungen aufnehmen können.

XLVII, 3 (März 1993), S. 218-230. Die Identifikation Rodós mit der Stimme Prósperos war noch immer eine Konstante der weltweit begangenen Feierlichkeiten im Centenario des Todesjahres Rodós.

16 Vgl. hierzu Real de Azúa, Carlos: Prólogo. In: Rodó, José Enrique: Ariel. Motivos de Proteo, S. X. 
Innenraum und Außenraum sind dadurch von Ebene zu Ebene aufeinander bezogen und nicht voneinander unabhängig. Somit ergeben sich Parallelen zwischen der expliziten räumlichen Strukturiertheit auf Inhaltsebene (Lehrsaal Großstadt - Kosmos) und der architextuellen Hierarchisierung auf Ausdrucksebene (Erzählung - Rede - fiktionaler Rahmen). Darüber hinaus ist eine mehrfach wiederkehrende Raumstruktur die der gotischen Kathedrale. Sie schließt sich im letzten Teil des siebten Kapitels unmittelbar an die eingangs zitierte Passage an und weist der Vision des kommenden Amerika einen bestimmten Ort zu: „Widmet ihm zumindest Eure Gedanken; die Ehre Eurer künftigen Geschichte hängt davon ab, ob Ihr beständig vor den Augen der Seele die Vision dieses erneuerten Amerika habt, die sich hoch über den gegenwärtigen Realitäten abzeichnet, der weiten Rosette eines gotischen Kirchenschiffs gleich, welche über der Strenge der düsteren Mauern im Lichte erglüht." ${ }^{17}$

Der Vergleich mit dem räumlichen Aufbau des gotischen Kirchenschiffs ist in mehrfacher Hinsicht bedeutsam; aber zwei Aspekte sind im Kontext finisekulärer Interieurs für unsere Fragestellung besonders wichtig: Zunächst zeigt sich in ihm die Sakralisierung der Vision des neuen Amerika; eine Vision, die auch auf einen menschlichen Innenraum bezogen wird, soll ihr Bild doch stets vor den „Augen der Seele" stehen. Die Verwendung dieser topischen Metapher ist dabei keineswegs beliebig; sie geht zurück auf Platons Politeia, wo sie in direkter Beziehung zur dialektischen Methode und zum Begriff der Idee steht, welche die geistige Gestalt der Dinge sichtbar macht. ${ }^{18}$ In ihrer langen Geschichte war die Metapher vom Auge der Seele seit jeher „unüberbietbarer sprachlicher Ausdruck für die Verbindung der Fähigkeiten des reichsten inneren und des reichsten äußeren Sinnes“19 und stand bei den spanischen Mystikern für die Gotteserfahrung der Seele ein. Bei

17 Rodó, José Enrique: Ariel, S. 179; vgl. Rodó, José Enrique: Ariel. Motivos de Proteo, S. 51: „Pensad en ella a lo menos; el honor de vuestra historia futura depende de que tengáis constantemente ante los ojos del alma la visión de esa América regenerada, cerniéndose de lo alto sobre las realidades del presente, como en la nave gótica el vasto rosetón que arde en la luz sobre lo austero de los muros sombríos.“

18 Vgl. hierzu die kurze Bemerkung von Curtius, Ernst Robert: Europäische Literatur und lateinisches Mittelalter. Bern - München: Francke Verlag ${ }^{10} 1984$, S. 146; vgl. Platon: Sämtliche Werke. Bd. 3: Phaidon, Politeia. In der Übersetzung von Friedrich Schleiermacher. Hamburg: Rowohlt 1958, S. 239.

19 Janik, Dieter: Das „Auge der Seele“: erkenntnistheoretische, religiöse, moralische und ästhetische Funktionen einer topischen Metapher ausgehend von Baltasar Gracián. In: Toro, Alfonso de (Hg.): Texte, Kontexte, Strukturen. Beiträge zur französischen, spanischen und hispanoamerikanischen Literatur. Festschrift zum 60. Geburtstag von Karl Alfred Blüher. Tübingen: Narr 1987, S. 372 . 
Santa Teresa etwa wird diese Metapher für die überraschende Einkehr Christi in die Seele des Menschen verwendet. ${ }^{20}$

Die „visión intelectual“ des kommenden, des in der menschlichen Seele Einkehr haltenden Gottes bleibt bei Santa Teresa den leiblichen Augen wie jenen der Seele verborgen. Erst die Worte des Meisters, und damit letztlich die Festigkeit des Glaubens, bringen in den „moradas sestas“ der ,inneren Burg“ die endgültige Gewissheit. Auch in Prósperos Rede ist nur die Zielvorstellung als Ideal, als Idee im Sinne Platons vor den Augen der Seele beständig. Die Frage, ob das Kind, das neue Amerika, der kommende Gott von den Schülern dereinst wahrgenommen werden kann, lässt sich daher nicht nur auf eine konkrete Zukunft beziehen, in der diese Vision vielleicht einmal in Wirklichkeit verwandelt sein wird; Prósperos rhetorische Frage meint zugleich auch die seelische, metaphysische, ja die mystische Dimension dieses Schauens. Auch beim Menschen sind in der Rede des Meisters Innen- und Außenraum, innere und äußere Sinne aufeinander bezogen. Wie in der Sakralisierung der Bronzestatue Ariels, der literarischen Figur aus Shakespeares The Tempest, zeigt sich erneut ein Spiel von Desakralisierung und Resakralisierung, das den gesamten Text Rodós durchzieht und mit dem von der Modernización ausgelösten Säkularisierungs-Prozess wie der gegenläufigen Bewegung einer Sakralisierung der Kunst in Verbindung zu bringen ist. Die literarische Figur Prósperos und eine die künftige Rezeption vorwegnehmende Wirkung seiner Worte auf die Schüler bezeichnen diesen neuen quasi-sakralen Raum der Literatur im Lateinamerika der Jahrhundertwende.

Der zweite Aspekt betrifft die Tatsache, dass auch in der gotischen Kathedrale der Innenraum in des Wortes ursprünglicher Bedeutung orientiert, also nach Osten ausgerichtet ist. Als Gegenpol zum Chor dient die Rosette, die ihr Licht nach Osten ins Kirchenschiff wirft: In ihrer Symbolik ist die Kathedrale Schiff und Wanderzelt, Altar und schwebende Stadt. ${ }^{21}$ Die Rosette schwebt als zentrierte und zentrierende Form über dem Dunkel auf einer anderen Ebene und symbolisiert die transzendente Öffnung auf einen mystischen Erfahrungsraum, der den Mikrokosmos Mensch in seinem Bezug zum Makrokosmos und in seiner göttlichen Eingebundenheit zeigt; eine Erfahrung, welche die Schüler Prósperos im letzten Kapitel unter dem Sternenzelt in geradezu ekstatischer Weise erleben.

20 Vgl. Ette, Ottmar: „La modernidad hospitalaria“: Santa Teresa, Rubén Darío y las dimensiones del espacio en „Ariel“ de José Enrique Rodó. In: Unión (La Habana) IX, 32 (julio - septiembre 1998), S. 2-11.

21 Zur Symbolik der gotischen Kathedrale vgl. Kunze, Konrad: Himmel in Stein. Freiburg Basel - Wien: Herder 1980. 
Auch der Platz unter den Sternen im Innenraum des gotischen Kirchenschiffs, der Chor, wird in Ariel evoziert. In explizitem Rückgriff auf einen Vergleich, den Marie Jean Guyau in Les problèmes de l'esthétique contemporaine (1884) benutzt hatte, dient das gotische Chorgestühl Próspero im vierten Kapitel dazu, die intime Verbindung zwischen Ethik und Ästhetik seinen Schülern vor Augen zu führen. Wir kommen nicht umhin, an dieser Stelle noch einmal auf die Interieurs in dem im selben Jahr erschienenen Roman von Joris-Karl Huysmans und auf die Innenarchitektur beim Tanz der Salomé zu verweisen. Der gotische Raum bei Rodó ist nicht mehr der einer Psychomachie, einer Opposition von Tugenden und Lastern, von Gut und Böse: Der räumliche Aufbau des Chorgestühls mit seiner Gegenüberstellung von Heiligenviten und Blumenornamenten verdeutlicht vielmehr die dialektische Überwindung einer Gegensätzlichkeit zwischen ästhetischer Schönheit und moralischer Wahrheit. Gewiss ist in Rodós Text die Ästhetik nicht mehr in der Ethik beziehungsweise im (christlichen) Glauben verankert, scheint doch in seinem Werk umgekehrt die Ethik eher in der Ästhetik fundiert zu sein. Wohl aber werden in der von Próspero unter Rückgriff auf Guyau entworfenen Raumstruktur ganz wie im gotischen Chorgestühl die gegenüberliegenden Pole aufeinander bezogen: So erst bilden sie einen integralen, ganzheitlichen Raum. Auf diese Weise entsteht ein Innenraum, der wiederum dem größeren Innenraum der Kathedrale zugeordnet ist, welcher seinerseits die Beziehung zum Außenraum des Kosmos symbolisiert. Diese Raumstruktur wird in Prósperos kurzem Dialog mit dem französischen Philosophen zum Sinnbild der Seele. ${ }^{22}$

Próspero selbst aber hatte zuvor, im dritten Kapitel, eine andere räumliche Struktur entworfen, die nach seinen Worten Symbol des Aufbaus der Seele ${ }^{23}$ sein sollte: die Burg des Gastfreundlichen Königs. Die architektonische Anlage dieses Alkazar ist literarisch im Orient verortet. ${ }^{24}$ In ihr vereinigen sich die offenen Formen orientalischer Paläste und Märkte mit jenen arabischer Wehrbauten, die jedoch durch breite, weit geöffnete und nicht bewachte Tore charakterisiert werden. Dieser Architektur des Orients werden offene Säulenhallen, die mehr an klassische griechische Formen erinnern, sowie einzelne Teile des Palastes entgegengestellt, welche als zunehmend von Pflanzen überwucherte Ruinen in Szene gesetzt werden. Die Mischung architektonischer Formen (und in der europäischen Romantik beheimateter literarischer Bilder) ist durch eine große Offenheit ausgezeichnet, gelangen doch Bettler zum Thron des Königs und Kinder

22 Rodó, José Enrique: Ariel. Motivos de Proteo, S. 18.

23 Ebda., S. 13: „Encuentro el símbolo de lo que debe ser nuestra alma en un cuento que evoco de un empolvado rincón de mi memoria.“

24 Ebda. 
allmorgendlich zu seinem Bett. Die Übergänge zwischen den Interieurs und den Außenräumen sind fließend. Selbst Pflanzen und Tieren steht der märchenhafte Palast offen, in dem eine paradiesische Harmonie zwischen allen Formen des Lebens zu herrschen scheint. Den offenen Raumstrukturen, die allen Menschen Einlass gewähren, vor allem aber seiner Großzügigkeit verdankt der patriarchalische Herrscher seinen Beinamen „el rey hospitalario“.

Dieser Offenheit steht jedoch die Verborgenheit und Verschlossenheit eines Raumes gegenüber, zu dem allein der König Zugang hat. Denn tief drinnen ${ }^{25}$ verwandelt sich die Architektur in eine labyrinthische Struktur verborgener Pfade, die zu einem Raum führen, welcher gänzlich vom lärmenden Alkazar durch dicke Porphyr-Quader abgeschirmt wird. Innenraum und Außenraum scheinen hier in einen unüberbrückbaren Gegensatz zu treten. Selbst das Licht vermag in diesen von „ernsten Karyatiden“ bewachten innersten Raum nicht einzudringen, der durch den Verweis auf Uhlands Gedicht Die verlorene Kirche in einen Zusammenhang mit der deutschen Romantik und deren Mittelalterbild gebracht wird. In dem von einer Artesonado-Decke überwölbten eigentlichen Zentrum der Palastanlage herrscht ein „,religiöses Schweigen“; doch endet die Gastfreundschaft des Königs hier nur scheinbar:

An den Stirnseiten sprachen in Stein gehauene Bilder von Idealität, von Selbstversunkenheit, von Ruhe ...- Und der alte König versicherte, daß seine Gastfreundschaft - dürfe ihn auch niemand je bis hierher begleiten - an dem rätselhaften Schutzorte so freigiebig und groß wie immer bliebe, nur daß jene, die er zwischen diesen schweigsamen Mauern um sich versammelte, flüchtige Gäste seien, die keine Hand zu berühren vermöge. An diesem Orte träumte der legendäre König, hier befreite er sich von der Wirklichkeit; hier wendeten sich seine Blicke nach innen [...]; niemand entweihte ihn, denn niemand hätte es gewagt, seinen Fuß respektlos dorthin zu setzen, wo der alte König mit seinen Träumen allein und abgeschieden in der Ultima Thule seiner Seele sein wollte.

Ich will, daß diese Geschichte in Eurem inneren Reiche den Ort ihrer Handlung finde. ${ }^{26}$

Prósperos sich unmittelbar anschließende Deutung dieses ,verbotenen“ Raums als Vorbedingung wahrer individueller Freiheit und seelisches Zentrum eines ganzheitlich gebildeten Menschen macht die Erzählung vom Gastfreundlichen König zu einer Parabel, die seiner Ansicht von der richtigen Entwicklung des menschlichen Wesens Ausdruck verleihen soll. Sie bezieht Offenheit (,a todas las corrientes del mundo“) wie Geschlossenheit gegenüber den „huéspedes profanos“ auf die Entwicklung des Individuums und weist damit der eingebet-

25 Ebda., S. 14: „dentro, muy dentro.“

26 Rodó, José Enrique: Ariel, S. 90. 
teten Gattung des „cuento“ eine eindeutige, illustrative Funktion innerhalb der Rede $\mathrm{zu}^{27}$

Die Parabel lässt sich aber auch auf den gesamten Text beziehen. Dies betrifft zunächst die auf der Inhaltsebene angesiedelte Raumstruktur: Die äußeren Bereiche der Palastarchitektur öffnen sich auf ihre Umgebung hin, sie gewähren Händlern wie Bauern Einlass. Auch die inneren Räume stehen nicht nur Tieren und Pflanzen, sondern auch Hilfesuchenden und Kindern offen. Der innerste Raum ist jedoch bewacht, ihn erreichen die profanen Gäste nicht mehr: Nur der Patriarch selbst und die „magnificencia de las sombras serenas“28 dürfen sich in diesem letzten Innenraum aufhalten, zu dem keine Schallwellen, nur noch wenige Lichtwellen, immerhin aber noch Düfte vordringen können. Es wiederholt sich damit die (dreistufige) Raumstruktur, die sich sowohl in der Anlage des Gesamttexts als auch in der Darstellung von Räumen in Prósperos Rede herausarbeiten ließ. Die im Fin de siècle hochgeschätzten Interieurs geben ein Bild vom Inneren des idealen Menschen.

Auch auf der Ausdrucksebene hatte unsere Analyse im Bereich des genretechnischen Aufbaus eine Anlage ergeben, die sich hinsichtlich ihrer räumlichen Strukturierung mit der Erzählung in Verbindung bringen lässt. Prósperos „cuento“, der sich unterhalb von fiktionalem Rahmen und Redestruktur auf einer dritten textinternen Ebene ansiedelt, erweist sich auch in dieser Beziehung als eine Mise en abyme des gesamten Textes. Dies verleiht der Frage nach dem literarischen Raum der Erzählung, mithin nach ihren intertextuellen Bezügen zu anderen Werken, ein noch größeres Gewicht. Widmen wir uns folglich nach dem architektonischen nun dem literarischen Raum!

Der sonst so freimütig auf seine Bezugstexte hinweisende Próspero gibt augenzwinkernd an, die Erzählung in einer „verstaubten Ecke“ seines Gedächtnisses gefunden $\mathrm{zu}$ haben. Vor einiger Zeit hat in recht unbestimmter Weise Roberto González Echevarría auf Bezüge zu den Moradas del castillo interior aufmerksam gemacht. ${ }^{29}$ Er brachte jedoch die in der Erzählung dargestellte Raumstruktur, die er als halb Palast, halb Bunker umschrieb, vorrangig mit Illustrationen von Texten Jules Vernes und mit jenen Diktatorenpalästen in Verbindung, welche die Architektur des lateinamerikanischen Diktatorenromans bestimmen. Doch scheint es mir geboten, den Beziehungen zur inneren Architektur der Seele, wie

27 Ebda., S. 15.

28 Ebda., S. 14.

29 Vgl. González Echevarría, Roberto: The Case of the Speaking Statue: „Ariel“ and the Magisterial Rhetoric of the Latin American Essay. In (ders.): The Voice of the Masters. Writing and Authority in Modern Latin American Literature. Austin: University of Texas Press 1985, S. 23. 
sie Santa Teresa in ihren Moradas entwarf, mit größerer Aufmerksamkeit nachzugehen. Próspero selbst weist gleich zu Beginn seiner Rede auf die „elfenbeinernen Verse“ der spanischen Mystiker hin. Er tut dies (erneut) in Zusammenhang mit einer Parabel Guyaus, welche nach Prósperos Worten das Hoffen der Menschheit mit den immer von neuem enttäuschten Erwartungen einer Braut vergleicht, die ihren künftigen Gatten empfangen will. Jeden Morgen legt sie - ein Opfer ihrer Träumereien und ihres Wahnsinns - aufs Neue ihren Brautschmuck an, in der Erwartung des Kommenden: „Es hoy cuando vendrä“, ${ }^{30}$ so die Formulierung, die nicht zufällig an Rodós 1897 erschienene Schrift El que vendrá erinnert. ${ }^{31}$ Próspero freilich zieht die Verbindung zu den Mystikern.

Die Einkehr des mystischen Gatten unterliegt in Santa Teresas Moradas nicht dem Willen des Menschen. Auch zeigt sich der ersehnte Gast in den „moradas sestas“ weder den Augen des Leibes noch den Augen der Seele. Ob die ersehnte Vereinigung mit dem Gatten, dem „Esposo“ jemals Wirklichkeit wird, ist ebenso ungewiss wie die Realisierung der Vision des kommenden Amerika, die der Seher Próspero seinen Schülern vor (die) Augen (der Seele) zu führen versucht. Was Santa Teresa widerstrebend als „visión intelectual“ bezeichnet, vermag die Fülle der Heilserfahrung jener „Braut Christi“ (hinter der sich gewiss auch die spanische Ordensfrau verbirgt) nicht zu erfassen, die sich trotz ihrer Verwirrung doch der Einkehr des Kommenden stets gewiss ist, auch wenn sie nicht wisse, wieso ihr so viel Glück beschieden ist.

Die Worte Teresas aus dem achten Kapitel der „moradas sestas“, die sich schon mit Prósperos Vision eines neuen Amerika in Verbindung bringen ließen, bilden eine zweite Lektüreebene seines Rückgriffs auf Guyaus Vergleich: Die Offenheit des Verweises auf die Bezugstexte täuscht. Denn das explizite Verweissystem in Ariel kaschiert ein implizites intertextuelles Beziehungsgeflecht. Seine Spuren werden an der Oberfläche durch die Verweise auf Autornamen verwischt, doch ist der Weg zu diesem inneren Verweissystem wie in der Burg des Gastfreundlichen Königs ein zwar verborgener, aber nicht unauffindbarer Pfad. Selbst auf lexikalischer Ebene lässt sich ein direkter Bezug zwischen den ,versos de marfil“ der Mystiker und der Erzählung vom Gastfreundlichen König herstellen, ist der innerste Raum im Palast des „rey hospitalario“ doch von Elfenbeintüren (,puertas de marfil“) verschlossen. Ihn öffnen gleichsam die Werke und Visionen der spanischen Mystik. So ist die Raumstruktur des Alkazar, der nach Prósperos Worten die Seele des Menschen symbolisiert, eng mit den Moradas del castillo

30 Rodó, José Enrique: Ariel. Motivos de Proteo, S. 5.

31 Eine noch deutlichere Verbindung findet sich in der Parallelstelle der ,invocación al ideal que vendrá, con una nota de esperanza mesiánica“ (Ebda., S. 8.). 
interior verknüpft, deren Anlage die innere Architektur der Seele und den Weg der Vervollkommnung, den Camino de Perfección einer Vereinigung mit dem Ideal, wie ihn auch die Schüler in Rodós Ariel durchlaufen, zu versinnbildlichen suchte. Auch der „rey hospitalario“ ist in diesem Innersten seiner Burg nur Gast. Erneut zeigt sich, diesmal auf intertextueller Ebene, ein (das gesamte 19. Jahrhundert durchlaufender) Prozess von De- und Resakralisierung bei der Anverwandlung der Texte mystischer Gottesschau: Denn aus der Unio mystica der spanischen Heiligen ist die ganzheitliche Gestaltung eines ideell ausgerichteten Lebens, zugleich aber auch die Vision einer künftigen Einheit Lateinamerikas geworden.

Unter dem expliziten Verweissystem des „cuento“, das den Orpheus-Mythos, die Werke des Heiligen Franz von Assisi und Die verlorene Kirche Uhlands aufruft, verbirgt sich ein implizites, das gleichsam den profanen Blicken entzogen ist. Auf dieser impliziten Ebene lassen sich jedoch nicht nur Verbindungen zu den spanischen Mystikern, sondern auch zu einem zeitgenössischen lateinamerikanischen Text herausarbeiten: Gemeint sind die Bezüge zwischen dem „rey hospitalario“ und Daríos Erzählung El rey burgués, einer der berühmtesten Erzählungen des nicaraguanischen Dichters, der neben Martí und Rodó die dritte der modernistischen „Tre Corone“ bildet.

In diesem „Cuento alegre“ (so der Untertitel) ${ }^{32}$ aus Daríos einflussreichem Prosabändchen Azul (1888) wird bewusst eine Spannung zwischen Rahmenstruktur und Binnenerzählung erzeugt, verspricht der Erzähler doch Zerstreuung und Erheiterung, die im Leser eine Erwartungshaltung provozieren, welche aber nachhaltig enttäuscht wird. Diese Ent-Täuschung hat in ganz wesentlicher Weise mit der poetologischen Dimension des „cuento“ zu tun, die im Vordergrund der hier vorgeschlagenen Lektüre stehen und auf Prósperos ,Märchen“ oder Parabel bezogen werden soll. Daríos Erzählung widersetzt sich der von außen an sie herangetragenen Zerstreuungsfunktion und macht in der vieldeutigen Auseinandersetzung zwischen Bürgerkönig und Dichter die Rolle des Schriftstellers in der bürgerlichen Gesellschaft zu einem zentralen Thema. ${ }^{33}$ Dabei wird des Bürgerkönigs ostentativer Gebrauch von Kunst ebenso ironisch in Szene gesetzt wie die wortreiche Anrufung des Ideals durch den Dichter, dessen Verse von Apotheker und Schuhmacher kritisiert werden. Doch auch am Hofe des Bürgerkönigs findet der Heimatlose keinen Mäzen, der ihm einen Freiraum für seine Kunst schaffen

$32 \mathrm{Zu}$ Beginn seiner Erzählung vom Rey Hospitalario hatte Próspero von der „alegre bandada de los cuentos“ (13, Hervorhebung O.E.) gesprochen - ein erster diskreter Verweis auf die intertextuelle Beziehung zu Daríos Erzählung.

33 Vgl. zum historischen Hintergrund dieser Thematik Real de Azúa, Carlos: Modernismo e ideologías. In: Punto de vista (Buenos Aires) IX, 28 (noviembre 1986), S. 1-42. 
könnte: Dem Ratschlag seines Philosophen folgend, verbannt der König den Poeten aus seinem Palast und weist ihm die Aufgabe zu, zur Zerstreuung der Gäste im Garten die Drehorgel zu bedienen; aus dem Dichter wird ein bestellter Leierkastenmann. Die Funktionslosigkeit der vom Poeten vorgetragenen Literaturkonzeption wird durch die Marginalisierung des Dichters und dessen Tod ebenso veranschaulicht wie durch den Sieg rivalisierender literarischer Ausdrucksformen, die wie Herrscherlob und bürgerliche Epopöe (der König ist ein begeisterter Leser der Romane des französischen Publizisten Georges Ohnet), Grammatik und Rhetorik allein bei Hofe Gefallen finden.

Die vom Philosophen angeregte Ausweisung des Poeten aus den Räumen des Palasts, die erkennbar auf Platons Verbannung der Dichter aus dem Staat anspielt, lenkt den Blick auf die Raumstruktur der Erzählung, die im Übrigen viele Elemente des so zeittypischen literarischen Orientalismus in sich aufnimmt. Der Palast ist in der Nähe einer modernen Großstadt angesiedelt und von Gärten umgeben. Wie im Alkazar des Gastfreundlichen Königs zeigt sich auch in ihm eine Mischung verschiedener Kulturen und kunstgeschichtlicher Epochen, sind seine prunkvollen Säle doch etwa der Kunst Asiens, der griechischen Antike oder dem französischen Rokoko gewidmet. Dabei ergibt sich freilich keine hierarchisch zentrierte, sondern eine additive Struktur:

Der König besaß einen glänzenden Palast, in welchem er Reichtümer und wunderbare Kunstobjekte angesammelt hatte. Er gelangte durch Lilienrabatten und ausgedehnte Teiche zu ihm, wobei er von den Schwänen mit ihren weißen Hälsen noch vor den verstreuten Lakaien gegrüßt wurde. Guter Geschmack. Er stieg über eine Treppe voller Alabaster- und Smaragdsäulen hinauf, an deren Seiten wie an Salomons Thronen Marmorlöwen wachten. Erlesenheit. Neben den Schwänen besaß er eine weite Vogel-Voliere, da er die Harmonien, das Gurren, das Geträller liebte, und ganz in ihrer Nähe ließ er seinem Geiste Auslauf, indem er Romane von Monsieur Ohnet oder schöne Bücher über Grammatikfragen oder ausgesuchte Kritiken las. Aber ja: ein entschlossener Verteidiger der akademischen Korrektheit in der Literatur und der Gelecktheit in den Künsten, eine sublime Seele, verliebt in Sandpapier und Orthographie! [...]

Im Übrigen besaß er einen griechischen Salon voller Marmorstücke: von Göttinnen, Musen, Nymphen und Satyrn; den Salon der galanten Zeiten mit Gemälden des großen Watteau und Chardin; zwei, drei, vier, wie viele Säle!

Und Maecenas durchschritt sie alle mit einem Gesicht, das von einer gewissen Majestät überflutet war, mit glücklichen Bauch und Krone auf dem Kopf, einem Spielkartenkönig gleich. ${ }^{34}$

34 Darío, Rubén: El rey burgués. In: ders.: Obras completas. Bd. V: Poesía. Madrid: Afrodisio Aguado 1953, S. $626 \mathrm{f}$. 
Mit der ruhelosen Bewegung des Bürgerkönigs durch seine verschiedenen, aber allesamt gleichwichtigen Säle kontrastiert die Ruhe des orientalischen Patriarchen, welche durch die Zentrierung seines Alkazar auf den innersten Raum erzeugt wird. Die Kunst am Hofe des Bürgerkönigs ist ostentativ und nach außen gerichtet, sie wird mit Hilfe von Fachwissen so akkumuliert, wie das Bürgertum Reichtum anhäuft; die Kunst am Hofe des Gastfreundlichen Königs hingegen ist spirituell und nach innen gerichtet, sie ordnet sich funktional einem Ideal von Ganzheit, von geistig-seelischer Synthese zu. Während sich die Gastfreundschaft von Rubén Daríos „rey burgués“ auf den gesamten Raum des Palastes zu beziehen und keine verbotenen Bereiche zu kennen scheint, erstreckt sich die Gastfreundschaft des „rey hospitalario“ in Rodós Ariel nicht auf alle Räume, sondern hält die profanen Gäste vom sakralen Innenraum fern. Während der „cuento alegre“ die Rivalität zwischen Philosoph und Dichter betont, sucht sich das bekannteste Werk des Uruguayers im Zwischenbereich zwischen Philosophie und Literatur - im Sinne einer „moderna literatura de ideas“35 - anzusiedeln. Auch in Rodós Ariel ist der Raum einer so verstandenen Literatur bedroht: Auch hier findet sich ein Apotheker, der mit Flauberts Zerrbild des erfolgreichen Apothekers Homais ${ }^{36}$ identifiziert wird und den Pol der Barbarei des Nützlichen in der modernen bürgerlichen Gesellschaft vertritt. Auch Prósperos „cuento“ kommt - gerade durch die versteckte Auseinandersetzung mit Daríos Text - eine poetologische Bedeutung zu, wird doch die von Daríos exiliertem Dichter ohnmächtig angerufene Idealität, der am Hofe des Bürgerkönigs kein Platz zugewiesen wird, ins Zentrum des innersten Raums der gastfreundlichen Burg gestellt. Die vom Poeten beschworene Ankunft eines lichtvollen und potenten Messias, eines „Mesías todo luz, todo agitación y potencia“"37 beziehungsweise die Präsenz des Poetischen „en el astro en el fondo del cielo“ und „en la perla en lo profundo del Océano“, ${ }^{38}$ sind Textelemente, die in Rodós Ariel in positiver Form wiederaufgenommen werden. Wie das messianische Element den gesamten Text durchzieht und Ariel mit dem Vibrieren der Sterne ausklingt, so öffnet sich der innerste Raum des Gastfreundlichen Königs in sternenklarer Nacht ganz wie eine Muschel.

Es wäre gewiss grundfalsch, aus Rodós positiver Aufnahme dieser romantischen Bilder und idealistischen Vorstellungen, welche Daríos Text unüber-

35 Rodó, José Enrique: Ariel. Motivos de Proteo, S. 31. Diesem Zitat aus Prósperos Rede lässt sich ein Brief Rodós vom 25.2.1901 an Miguel de Unamuno, wo der uruguayische Literat ebenfalls von einer ,literatura de ideas“ spricht, an die Seite stellen; vgl. Rodó, José Enrique: Obras Completas, S. 1308.

36 Rodó, José Enrique: Ariel. Motivos de Proteo, S. 26.

37 Darío, Rubén: El rey burgués, S. 629.

38 Ebda. 
hörbar ironisch präsentiert, eine Rückkehr des Uruguayers zu Positionen einer lateinamerikanischen oder europäischen Romantik ableiten zu wollen. Ebenso falsch wäre es, in dieser Erzählung eine literarische Evasion angesichts jener Veränderungen zu erblicken, die der Modernisierungsprozess in Bezug auf die Struktur der gesamten Gesellschaft, aber auch auf die soziale Rolle des Künstlers in der Moderne ausgelöst hatte. Doch weisen diese Textelemente auf eine vor allem im „cuento“ vom Gastfreundlichen König vorgenommene immanente poetologische Auseinandersetzung mit Darío, wie sie in einem Artikel von 1897, ${ }^{39}$ vor allem aber in Rodós 1899 veröffentlichter Studie über den Nicaraguaner explizit vorgenommen wurde. In seinem ein Jahr vor Ariel veröffentlichten Essay Rubén Dario. Su personalidad literaria, su última obra hatte der junge Rodó nicht nur sein modernistisches Credo abgelegt, sondern zugleich versucht, seinen eigenen Standort innerhalb des Modernismo zu definieren. Dabei setzte er sich nicht nur von dem an Darío ausgerichteten, mit modernistischen Farbenspielen infantil beschäftigten Epigonentum $\mathrm{ab},{ }^{40}$ sondern bestimmte auch kritisch seine eigene Position gegenüber dem damals bereits einflussreichsten und bekanntesten Vertreter des hispanoamerikanischen Modernismus. Darío besitze eine machtvolle dichterische Individualität, doch gepaart mit einer nicht weniger poetischen Verantwortungslosigkeit. ${ }^{41}$

Rodós Bekenntnis zum Modernismo, verbunden mit einer subtilen Kritik am Schreiben des Nicaraguaners, brachte seine Infragestellung einer Darío-zentrischen Sichtweise des Modernismus zum Ausdruck, welche die Beziehungen zwischen beiden Autoren nachhaltig belastete und zu einer Reihe versteckter Auseinandersetzungen führen sollte. Darío übernahm zwar Rodós Studie mit dessen Genehmigung als Vorwort für die zweite Auflage der Prosas profanas, ,vergaß“ aber, den Namen des Verfassers mitabdrucken zu lassen, was die Irritationen weiter verstärkte. Noch das autobiographische Eröffnungsgedicht Yo soy aquel in Daríos Cantos de vida y esperanza lässt sich als poetologische Auseinandersetzung mit der Kritik des Uruguayers lesen. ${ }^{42}$ Als „buenos camaradas de ideas“ seien beide Modernisten dazu berufen, die Konzeptionen von Naturalismus und

39 „A Rubén Darío le está permitido emanciparse de la obligación humana de la lucha, refugiarse en el Oriente o en Grecia, madrigalizar con los abates galantes, hacer la corte a las marquesas de Watteau naturalizándose en el ,país‘ donoso de los abanicos.“ Rodó, José Enrique: Un poeta en Caracas. In (ders.): Obras Completas, S. 847.

40 Rodó, José Enrique: Rubén Darío. Su personalidad literaria, su última obra, S. 187.

41 Ebda.

42 Vgl. hierzu Molloy, Sylvia: Ser / decir: tácticas de un autorretrato. In: Fernández Cifuentes, L. / Molloy, Sylvia (Hg.): Essays on Hispanic Literature in Honor of Edmund L. King. London: Tamesis Books 1983, S. 187-199. Auf andere Bezüge zwischen Darío und Rodó macht aufmerksam 
Positivismus in Zeiten epochaler Veränderungen in größere ästhetische Höhen zu heben: „Auch ich bin ein Modernist“, affirmierte Rodó entschieden. ${ }^{43}$

José Enrique Rodó verwirklichte seine Konzeption einer „modernen Ideenliteratur“, die im Gegensatz zu seiner Ansicht von Daríos ästhetischer Praxis Denken und Fühlen miteinander vereinigen sollte, mit Ariel ein erstes Mal in überzeugender Weise. Die Parabel vom Gastfreundlichen König lässt sich dabei als immanente Poetik und versteckte kreative Auseinandersetzung mit Daríos El rey burgués wie mit der Ästhetik des Dichters der Prosas profanas insgesamt deuten. Die Beziehung des „rey hospitalario“ zu Rodós Beschäftigung mit Darío besitzt dabei nicht nur einen intratextuellen, sondern sogar einen kotextuellen Status: Ariel wurde 1900 als dritter Teil des Zyklus La Vida Nueva veröffentlicht; in dessen erstem Teil war 1897 El que vendrá publiziert worden, bevor 1899 der zweite Teil von La Vida Nueva unter dem Titel Rubén Darío erschien. So zeigt sich, dass die Erzählung vom Gastfreundlichen König nicht nur auf inhaltlicher und architextueller Ebene eine Mise en abyme der zentrierten Raumstrukturen in Ariel bildet. Sie erfüllt diese Funktion auch auf der poetologischen und intertextuellen Ebene, indem sie einerseits eine Bestimmung des eigenen Schreibens innerhalb des Modernismo darstellt und andererseits eine intertextuelle Raumstruktur entwirft. Der innerliterarische Raum von Rodós Ariel ist durch ein explizites Verweissystem charakterisiert, unterhalb dessen sich ein implizites, verborgenes Bezugssystem artikuliert, dem eine enorme Bedeutung für das Verständnis des gesamten Textes zukommt. Auf diese Weise schreibt sich Ariel in den literarischen Bewegungsraum zwischen zwei Welten sowie ganz bewusst in die damals noch funktionierenden Parameter einer Weltliteratur ein.

Die lateinamerikanischen Literaturen siedeln sich innerhalb eines Spannungsfeldes an, das schematisch von den sechs Polen der abendländischen Kulturtraditionen, der indianischen Kulturen, der schwarzen Kulturen, der iberischen beziehungsweise europäischen Volkskulturen sowie transkulturellen oder hybriden Formen als Ergebnis des Kontakts zwischen den zuvor genannten Polen und schließlich einer globalisierten Massenkultur im Kontext modernisierter Kommunikationsnetze gebildet wird. Rodós Ariel orientiert sich von diesem Schema ausgehend ausschließlich am erstgenannten Pol. Darin fällt er deutlich hinter die umfassenden kulturellen Konzeptionen eines José Martí zurück. In Ariel rekurriert das in Prósperos Rede entworfene Bildungs- und Zivilisationsideal insbesondere auf die heidnische Antike und die christliche Heilslehre, ein Rückgriff,

Arellano, Jorge Eduardo: „Azul ...“ de Rubén Darío. Nuevas perspectivas. Washington: OEA / OAS 1993, S. $11 \mathrm{f}$. und $48 \mathrm{f}$.

43 Rodó, José Enrique: Rubén Darío, S. 52 f. 
der sich ideologisch an den Prämissen des von Michel Chevalier oder der Revue des races latines vertretenen Panlatinismus orientiert. Selbst die kulturelle Alterität wird nicht etwa aus den in Lateinamerika heimischen nicht-okzidentalen Kulturen oder aus der im Sinne Martís eigenen amerikanischen Antike bezogen, sondern erscheint im Gewand des literarischen Orientalismus, mit dem Europa im 19. Jahrhundert das kulturell Andere ,verkleidet‘ hatte.

Dominiert auf der Ebene des expliziten Verweissystems in Rodós Ariel auch deutlich die Orientierung an Frankreich, die etwa in der Erwähnung von Renan, Hugo, Guyau, Chevalier oder Fouillée zum Ausdruck kommt, so wäre es angesichts der Vielzahl von Verweisen auf englische wie deutsche, aber auch USamerikanische Literatur und Philosophie verfehlt, von einer einseitigen Ausrichtung zu sprechen. ${ }^{44}$ Schon Leopoldo Alas hatte in seiner 1900 verfassten Besprechung Ariels keineswegs den von ihm so oft geäußerten Vorwurf eines „galicismo mental“ gegen den uruguayischen Autor erhoben, sondern vielmehr auf Rodós vitale Verbindung zu Spanien verwiesen. Er tat dies mit Recht, wie die zwar implizite, aber intensive intertextuelle Beziehung zu Santa Teresas Moradas del castillo interior zeigte. Auch Rodós geschickte Strategie, den Text des spanischen Kritikers und Romanciers ab der zweiten, noch im Jahr 1900 in Montevideo erschienenen Ausgabe seinem Werk als Vorwort voranzustellen, mag darauf verweisen, wie wichtig es ihm gerade im Kontext der Ereignisse von 1898, angesichts der imperialistischen Ausdehnung der USA war, die transatlantisch-hispanische Traditionslinie zu betonen. Denn die Verwandlung des Textes von Leopoldo Alas in einen Paratext des eigenen Werks verlieh Ariel nicht nur höheres internationales Prestige und eine bessere Ausgangsposition für die Rezeption des Bandes in Spanien, sondern gab Rodó auch die Möglichkeit, ebenso subtil wie indirekt auf die Präsenz der hispanischen Literaturtradition in seinem Werk aufmerksam zu machen.

In Amerika begibt sich die ,gastfreundliche Moderne' nicht mehr nach Europa, sondern holt die Moderne nach Amerika. Diese Bewegung verläuft parallel zur Richtung der Immigration, die in Prósperos eingangs zitierter Vision eines gastfreundlichen Amerika in Erscheinung tritt. Dabei erstreckt sich wie bei der gerade am Río de la Plata staatlich geförderten Einwanderung die Gastfreundschaft keineswegs auf alle: So wie man vorrangig nur die Europäer willkommen hieß, beschränkte sich auch die Gastfreundschaft in Rodós Ariel auf den ersten, den abendländischen beziehungsweise europäischen Pol. Calibán, Sohn der ein-

44 Diesen in der Rodó-Rezeption traditionsreichen Vorwurf erhob zuletzt Carlos Fuentes in seinem Vorwort zu einer englischsprachigen Neuausgabe von Ariel; vgl. Fuentes, Carlos: Prologue. In: Rodó, José Enrique: Ariel. Austin: University of Texas Press 1988, S. 13-28. 
heimischen Hexe Sycorax und ihrer Kultur, ist nicht zum Gastmahl, nicht zum Dialog mit dem Meister geladen: Er bleibt draußen vor der Tür!

Rodós Ariel hatte schon bald riesigen Erfolg in ganz Lateinamerika. Die Zurechnung des Texts zur Gattung Essay schloss die Stimme Prósperos mit der Stimme seines Meisters, des uruguayischen Autors kurz. Rodós nur auf den ersten Blick dualistische Gegenüberstellung von arielistischem Lateinamerika und calibanesken USA war innerhalb des zeitgeschichtlichen Kontexts der nordamerikanischen Expansion opportun: So wurde Ariel als Pamphlet gelesen und auf das den USA gewidmete Kapitel reduziert. An der Verwechslung Prósperos mit Rodó war letzterer gewiss nicht unschuldig, griff er doch in seiner umfangreichen Korrespondenz gerne auf Formulierungen zurück, die er seiner literarischen Figur in den Mund gelegt hatte. Textexterne wie textinterne Faktoren trugen so dazu bei, dass die Komplexität des literarischen Aufbaus zugunsten einer raschen politischen Verwertbarkeit (durch links- wie rechtsarielistische Positionen) negiert werden konnte.

Doch Ariel ist kein Pamphlet; und die zahlreichen Feierlichkeiten und Tagungen aus Anlass seines hundertsten Todestages zeigen, dass Rodó und sein bekanntestes Werk heute keineswegs vergessen sind. Seine Deutung des Modernisierungsprozesses und sein Entwurf einer Literatur, die der Moderne adäquat sein will, zeigen deutlich den Versuch, die Moderne aus der Perspektive eines lateinischen Amerika zu denken. In diesem Sinne ist das „Así habló Próspero“, ${ }^{45}$ sein „Also sprach Próspero“, eine selbstbewusste Replik auf die Reden Zarathustras und damit auf einen großen Denker des europäischen Fin de siècle. Bereits in diesem kurzen Satz kommen Anverwandlung und Umwandlung einer europäischen Tradition im lateinischen Amerika deutlich zum Ausdruck:

Innerhalb der zeitgenössischen Literatur des Nordens, in der die Sorge um die hehren gesellschaftlichen Fragen so lebendig ist, taucht häufig der Ausdruck derselben Idee, desselben Gefühls auf; Ibsen entwickelt die stolze Ansprache seines Stockmann ausgehend von der Behauptung, daß „die kompakten Mehrheiten der gefährlichste Feind für Freiheit und Wahrheit sind“; und der ungeheure Nietzsche stellt dem Ideal einer Menschheit des Mittelmaßes die Apotheose der Seelen entgegen, die sich über das Niveau der Menschheit wie eine lebendige Flutwelle erheben. ${ }^{46}$

Die Wichtigkeit von Friedrich Nietzsche für den Autor von Ariel kann gar nicht überschätzt werden! Auf seiner Suche nach einer „modernen Ideenliteratur“ war ihm der deutsche Philosoph und Philologe eine wichtige Hilfe, hatte Nietzsche

45 Rodó, José Enrique: Ariel. Motivos de Proteo, S. 55.

46 Rodó, José Enrique: Ariel, S. 124. 
doch auf grundlegende Weise den Unterschied zwischen Literatur und Philosophie unterlaufen und damit für eine Ideen-Literatur Pate gestanden, wie Rodó sie im Sinne hatte. ${ }^{47}$ Der Rückgriff auf den „formidable Nietzsche“ erlaubte es Rodó überdies, dem Modernismo in Abgrenzung zu Rubén Darío eine stärker philosophische Ausrichtung zu geben, was seinen eigenen Schreibmöglichkeiten und Erwartungen jenseits der poetischen Potenz eines Darío Auftrieb verlieh. Dabei warnte Rodó zugleich vor dem „,anti-igualitarismo de Nietzsche“ ${ }^{48}$ der ihm durchaus suspekt war; dies hinderte ihn freilich nicht daran, sich das nietzscheanische Schreiben als Bezugsmodell zu wählen. Keine fünfzehn Jahre vor der Veröffentlichung von Ariel hatte der Verfasser von Jenseits von Gut und Böse zwischen verschiedenen ,rassischen“ und ,religiösen“ Blöcken unterschieden: „Es scheint, dass den lateinischen Rassen ihr Katholicismus viel innerlicher zugehört als uns Nordländern das ganze Christentum überhaupt: und dass folglich der Unglaube in katholischen Ländern etwas ganz Anderes zu bedeuten hat, als in protestantischen - nämlich eine Art Empörung gegen den Geist der Rasse, während er bei uns eher eine Rückkehr zum Geist (oder Ungeist -) der Rasse ist. “49

Wenn wir uns dies vor Augen halten, dann erscheint Rodós Werk in vielerlei Hinsicht als ein Versuch, gegen das Schaffen Friedrich Nietzsches einen lateinischen Gegenpol zu schaffen. Ariel erscheint so als eine Art ,Anti-Zarathustra', geschrieben von einem amerikanischen Vertreter eines Panlatinismus, der freilich für die Strömungen des ,Nordens“ offen war. So hatte Próspero vehement die Grundlagen des Christentums, gleichsam den Génie du Christianisme vertreten und war den Spuren des Saint Paul sowie vor allem der Vie de Jésus von Ernest Renan gefolgt; zwei Bände, die man später in der Bibliothek von Rodó fand. Der Verfasser der Motivos de Proteo dürfte dabei sehr wohl gewusst haben, dass Nietzsche in Der Antichrist Renan vehement angegriffen und ihn als „Herr Renan, dieser Hanswurst in psychologicis“, ${ }^{50}$ verspottet hatte.

Doch dem Autor von El que vendrá war zweifellos ebenso bewusst, dass Nietzsche in Jenseits von Gut und Böse „die kommenden“ erwähnt hatte, „die neuen

47 Zur Beziehung zwischen Rodío und Nietzsche vgl. ausführlich Ette, Ottmar: „Así habló Próspero“. Nietzsche, Rodó y la modernidad filosófica de „Ariel“. In: Cuadernos Hispanoamericanos (Madrid) 528 (junio 1994), S. 48-62.

48 Rodó, José Enrique: Ariel. Motivos de Proteo, S. 31.

49 Nietzsche, Friedrich: Jenseits von Gut und Böse. In (ders.): Werke. Kritische Gesamtausgabe. Ed. Giogio Colli y Mazzino Montinari. 6. Abt., 2. Band. Berlin: Walter de Gruyter 1968, S. 67.

50 Nietzsche, Friedrich: Der Antichrist. In (ders.): Werke. 6. Abt., 3. Bd.: Der Fall Wagner. GötzenDämmerung. Der Antichrist. Ecce homo. Dionysos-Dithyramben. Nietzsche contra Wagner. Berlin: Walter de Gruyter 1969, S. 197. 
Philosophen“; ${ }^{51}$ eine Vorstellung, die er in Ariel intensivierte, um zugleich die Beziehungen zu Nietzsches Zarathustra als einer Figur zu vervielfachen, welche wie Shakespeares Ariel ebenfalls beflügelt und mit Flügeln ausgestattet ist: „Zarathustra der Tänzer, Zarathustra der Leichte, der mit den Flügeln winkt, ein Flugbereiter, allen Vögeln zuwinkend, bereit und fertig, ein Selig-Leichtfertiger: Zarathustra der Wahrsager, Zarathustra der Wahrlacher, kein Ungeduldiger, kein Unbedingter, Einer, der Sprünge und Seitensprünge liebt [...].“52

Die Figur von Shakespeares „airy spirit“ entspricht in vielem dem, was Nietzsche das Apollinische nannte. Die zentrierten Raumstrukturen Ariels, in deren Mittelpunkt immer die Herrschaft des Geistigen, Spirituellen, Ideellen steht, weisen der Literatur einen ideellen Raum zu, in welchem sich Shakespeare, Nietzsche und Santa Teresa miteinander verbinden. Nicht von ungefähr wird die Verzückung der Schüler im achten und letzten Kapitel mit dem Begriff des „recogimiento“ eingeleitet, der für zentrale spirituelle Erfahrungen (und eine bestimmte Methode meditativer Gottesschau) im Werk der spanischen Mystikerin steht. Zugleich hatte Rubén Daríos „cuento alegre“ die Gefahr des Funktionsverlusts von Literatur in einer säkularisierten und modernisierten bürgerlichen Gesellschaft deutlich aufgezeigt. Innerhalb einer solchen weist Rodó der Literatur einen eigenen Ort, einen quasi-sakralen Bereich zu, von dem aus sie ihren Führungsanspruch geltend machen kann, denn:

\begin{abstract}
Also sprach Prospero.- Die jungen Schüler trennten sich vom Meister, nachdem sie ihm wie Söhne voller Zuneigung die Hand gedrückt hatten. Von seinem sanften Worte begleitete sie das anhaltende Vibrieren, das den Klagelaut eines angeschlagenen Kristallglases in heiterer Atmosphäre ausklingen lässt. Es war die letzte Stunde des Nachmittags. Ein Lichtstrahl der ersterbenden Sonne querte das verschwiegene Halbdunkel des Raumes und schien, die Stirn der Bronzestatue berührend, in den stolzen Augen Ariels den unruhigen Funken des Lebens zu entfachen. Sich weiter verlängernd, erinnerte der Lichtstrahl an einen langen Blick, den der Geist, gefangen in seiner Bronze, auf die sich entfernende jugendliche Gruppe warf. ${ }^{53}$
\end{abstract}

Die Literatur im Sinne Rodós übernimmt ihren führenden, quasi sakralen Anspruch und ihre Rolle von der Generation Sarmientos und Alberdis, die in Ariel teilweise mit Zitaten präsent, wenn auch nicht namentlich genannt ist. Im Unter-

51 Nietzsche, Friedrich: Werke. Kritische Gesamtausgabe. Ed. Giogio Colli und Mazzino Montinari. 6. Abteilung, 2. Bd: Jenseits von Gut und Böse. Zur Genealogie der Moral (1886-1887). Berlin: Walter de Gruyter 1968, S. 59.

52 Nietzsche, Friedrich: Also sprach Zarathustra. In (ders.): Werke. 6. Abt., 1. Bd.: Also sprach Zarathustra. Ein Buch für Alle und Keinen (1883-1885). Berlin: Walter de Gruyter 1968, S. 362. 53 Rodó, José Enrique: Ariel, S. 189. 
schied zu dieser dem Romanticismo zuzurechnenden Generation von Politikern und Literaten erhebt sie an der Jahrhundertwende ihren Führungsanspruch von einem eigenen Raum aus, der profanen Gästen nicht zugänglich ist. Der innerste Raum der ,inneren Burg“ im Palast des orientalischen Patriarchen wie das Chorgestühl innerhalb der gotischen Kathedrale weisen beide die gleiche Modellierung eines sakralisierten Interieurs auf, das finisekulären Zuschnitts ist. Beide literarischen Raumentwürfe sind in der für Prósperos Rede grundlegenden Bewegung vom Individuellen zum Kollektiven nicht nur als Sinnbilder der menschlichen Seele, sondern als Projektionen einer künftigen Gesellschaft in Lateinamerika zu verstehen.

In Ariel kulminiert ein 19. Jahrhundert zwischen zwei Welten, das im Geiste des Panlatinismus die transatlantischen Beziehungen ins Zentrum einer Modernisierung stellte, die sich auf das folgende 20. Jahrhundert öffnete - ohne sich auch nur vorstellen zu können, dass für die meisten Länder Lateinamerikas dieses neue Jahrhundert im gesellschaftspolitischen und sozioökonomischen Sinne ein verlorenes sein würde. Nicht aber im literarischen: Denn der Modernismo öffnete die Türen für jene Entwicklungen der lateinamerikanischen Literaturen, welche die transatlantischen Asymmetrien grundlegend verändern sollten. Der Shakespeare'sche Luftgeist Ariel hat die Stimmungen des Fin de siècle abgeschüttelt und weist voraus in ein neues Jahrhundert, in welches er gleichwohl vieles vom vergangenen Jahrhundert mit sich hinüberschleppt. In Ariel finden sich die Ideenwelten eines transarealen 19. Jahrhunderts, die sich von Chateaubriands Génie du Christianisme bis in das christliche Bekenntnis eines Joris-Karl Huysmans ziehen, aber zugleich Reflexionen einer sich verschiedenen Modernisierungsschüben stellenden Gesellschaft - Überlegungen, die von Germaine de Staël über Alexis de Tocqueville bis zu José Martís Nuestra América reichen. Rodó hat versucht, Bilanz zu ziehen und neue Ausblicke zu schaffen. In seinem Ariel treffen sich Shakespeare, Santa Teresa und Nietzsche zu einem gemeinsamen Diskurs, der für die Romanischen Literaturen der Welt eine neue, eine eigene Moderne und damit eine neue Zukunft ersehnt. Von der untergehenden Sonne, von Westen also erhält Ariels Statue im achten und letzten Kapitel jenen Lichtstrahl, der die künstlerische Form lebendig werden lässt und in ihr „la chispa inquieta de la vida“, ${ }^{4}$ den unruhigen und zugleich unendlichen Funken des Lebens entfacht. Dieser Sonnenuntergang ist kein Ende, sondern ein neuer Anfang. 
